\title{
SEMILEPTONIC $D$ DECAYS FROM CLEO AND BELLE
}

\author{
YONGSHENG GAO \\ Physics Department, Southern Methodist University, Dallas, TX 75275, USA
}

\begin{abstract}
Recent semileptonic $D$ meson decay results from CLEO and BELLE are summarized, including the improved measurements of absolute branching fractions for exclusive $D^{0}$ semileptonic decays into $K^{-} e^{+} \nu, \pi^{-} e^{+} \nu$ and $K^{*-} e^{+} \nu$, and the first observation and absolute branching fraction measurement of $D^{0} \rightarrow \rho^{-} e^{+} \nu$ with the first CLEO-c data sample.
\end{abstract}

\section{Introduction}

Semileptonic $D$ meson decays are of great physics interest because their description is relatively simple. The decay matrix for semileptonic $D$ meson decay decouples into a weak current component (describing the $W \ell \nu_{\ell}$ vertex), and a strong current term (for the $W c \bar{q}$ vertex) that is parameterized through form factor functions of the invariant mass $\left(q^{2}\right)$ of the $W$ exchanged. The form factors can not be easily computed in quantum chromodynamics (QCD) since they are affected by significant nonperturbative contributions. That is the main source of uncertainty in the extraction of the CKM matrix element from the simple decay processes. Precise experimental measurements are needed to guide theoretical progress in this area. Charm semileptonic decays allow a measurement of the form factors and CKM matrix elements $V_{c s}$ and $V_{c d}$. Using Heavy Quark Effective Theory (HQET) or lattice gauge techniques, the measured charm form factor can be related to those needed to interpret $b \rightarrow u$ transition and the measurement of the CKM matrix element $V_{u b}$.

While measurements of form factors in all charm exclusive semileptonic decays are important, those in pseudoscalar-topseudoscalar transitions are the easiest to perform. The differential decay rate for the exclusive semileptonic decay $D \rightarrow P \ell \nu(P$ stands for a pseudoscalar meson) with the electron mass effects neglected can be ex-

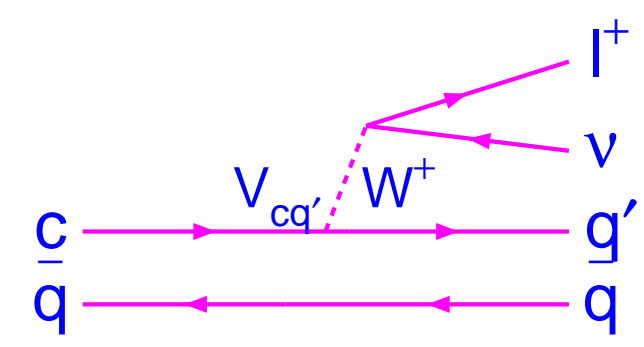

Figure 1. Feynman diagram for charm meson semileptonic decays.

pressed as ${ }^{1}$ :

$$
\frac{d \Gamma}{d q^{2}}=\frac{G_{F}^{2}}{24 \pi^{3}}\left|V_{c q^{\prime}}\right|^{2} p_{P}^{3}\left|f_{+}\left(q^{2}\right)\right|^{2}
$$

where $G_{F}$ is the Fermi coupling constant, $q^{2}$ is the four-momentum transfer squared between the parent $D$ meson and the final state meson, $p_{P}$ is the momentum of the pseudoscalar meson in the $D$ rest frame, and $V_{c q^{\prime}}$ is the relevant CKM matrix element, either $V_{c s}$ or $V_{c d}$. $f_{+}\left(q^{2}\right)$ is the form factor that measures the probability that the flavor changed quark $q^{\prime}$ and the spectator quark $\bar{q}$ in Figure 1 will form a meson in the final state.

Because the semileptonic $D$ meson decay results from BELLE were not available by ICHEP04, I'll only describe the recent work and results on this topic from CLEO. 
$2 \quad D^{0} \rightarrow \pi^{-} \ell^{+} \nu$ and $D^{0} \rightarrow K^{-} \ell^{+} \nu$

from CLEO III

We present a study of the decay $D^{0} \rightarrow \pi^{-} \ell^{+} \nu$ and $D^{0} \rightarrow K^{-} \ell^{+} \nu$ where $\ell=e$ or $\mu$ with $e^{+} e^{-} \rightarrow c \bar{c}$ events collected at and just below the $\Upsilon(4 \mathrm{~S})$ resonance with the CLEO III detector. We use only runs with good lepton identification, which leads to slightly different, but overlapping, datasets for the electron and muon modes with integrated luminosities of 6.7 and $8.0 \mathrm{fb}^{-1}$ respectively.

We use the decay chain of $D^{*+} \rightarrow D^{0} \pi^{+}$, and $D^{0}$ further decays to $\pi \ell \nu$ or $K \ell \nu$. The slow charged pion helps the identification and background rejection for $D^{0}$. The experimental observable is $\Delta M$ which is the mass difference of $D^{*+}$ and $D^{0} . D^{0}$ candidates are reconstructed from lepton, hadron ( $\pi$ or $K$ ), and neutrino combinations. A major challenge is the contamination of the Cabibbo-suppressed $D^{0} \rightarrow \pi^{-} \ell^{+} \nu$ sample by the Cabibbo-favored $D^{0} \rightarrow K^{-} \ell^{+} \nu$ decays, which are about a factor of 10 more common. The use of a Ring Imaging Cherenkov (RICH) detector and specific ionization in the drift chamber $(d E / d x)$ reduces this contamination dramatically by distinguishing $K$ from $\pi$ mesons. The resulting efficiency and misidentification probability suppress misidentified $D^{0} \rightarrow K^{-} \ell^{+} \nu$ decays to $15 \%$ of the $D^{0} \rightarrow \pi^{-} \ell^{+} \nu$ signal. Detailed event selection can be found in ${ }^{2}$.

Figure 2 shows the fits to the $\Delta M$ distributions for $D^{0} \rightarrow K^{-} \ell^{+} \nu$ and $D^{0} \rightarrow \pi^{-} \ell^{+} \nu$ and their confidence levels (C.L.). We divide the data into three $q^{2}$ bins. The bin size is guided by our $q^{2}$ resolution of $0.4 \mathrm{GeV}^{2}$. We measure the ratio of the branching fractions, $R_{0}=\mathcal{B}\left(D^{0} \rightarrow \pi^{-} \ell^{+} \nu\right) / \mathcal{B}\left(D^{0} \rightarrow K^{-} \ell^{+} \nu\right)$ to be $R_{0}=0.082 \pm 0.006 \pm 0.005$. This result is consistent with the previous world average of $0.101 \pm 0.018^{3}$ but more precise. We then use a simple pole parameterization to determine parameters describing the form factors by fitting the corrected $q^{2}$ distribu-

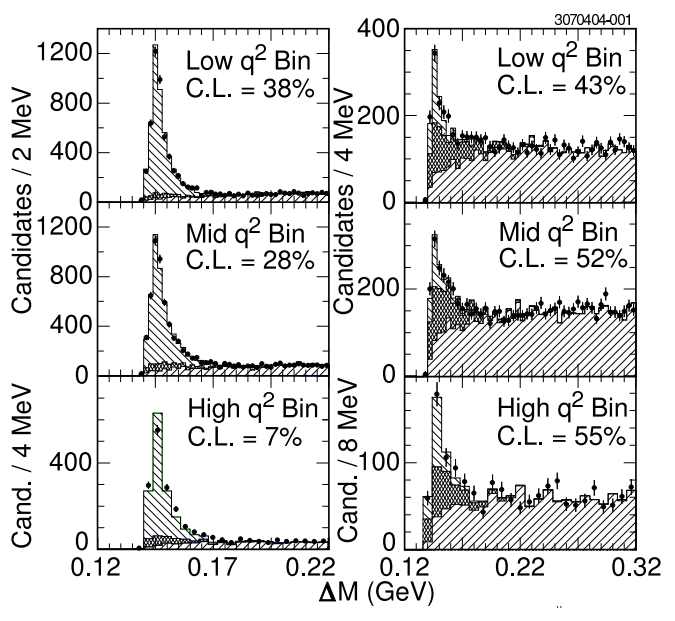

Figure 2. The fits to the $\Delta M$ distributions for $D^{0} \rightarrow$ $K^{-} \ell^{+} \nu$ (left) and $D^{0} \rightarrow \pi^{-} \ell^{+} \nu$ (right) and their confidence levels (C.L.). The data (points) are superimposed on the sum of the normalized simulated signal (peaked histogram), peaking background (dark histogram) and false- $\pi_{s}$ background (broad histogram).

tions. We find $\left|f_{+}^{\pi}(0)\right|^{2}\left|V_{c d}\right|^{2} /\left|f_{+}^{K}(0)\right|^{2}\left|V_{c s}\right|^{2}$ $=0.038_{-0.007-0.003}^{+0.006+0.005}$. Using $\left|V_{c d} / V_{c s}\right|^{2}$ $=0.052 \pm 0.001^{3}$ gives $\left|f_{+}^{\pi}(0)\right| /\left|f_{+}^{K}(0)\right|=$ $0.86 \pm 0.07_{-0.04}^{+0.06} \pm 0.01$. The details of this work are described in ${ }^{2}$ and have been submitted to PRL.

\section{First CLEO-c results on exclusive $D^{0}$ Semileptonic Decays}

Just like $\Upsilon(4 \mathrm{~S})$ which is an ideal laboratory for $B$ physics study at $e^{+} e^{-}$collider, $\Psi(3770)$ offers many advantages for Charm physics than $\Upsilon(4 \mathrm{~S})$. The advantages come from the threshold production of charm and the unique kinematics constrains associated with threshold production. The data sample used for this analysis was collected at the $\psi(3770)$ resonance with the CLEO-c detector. It corresponds to an integrated luminosity of $60 \mathrm{pb}^{-1}$.

We first select events with a fully reconstructed $D^{0}$ meson where $D^{0} \rightarrow K^{-} \pi^{+}$, $K^{-} \pi^{+} \pi^{0}, K^{-} \pi^{+} \pi^{0} \pi^{0}, K^{-} \pi^{+} \pi^{+} \pi^{-}, K_{S} \pi^{0}$, 


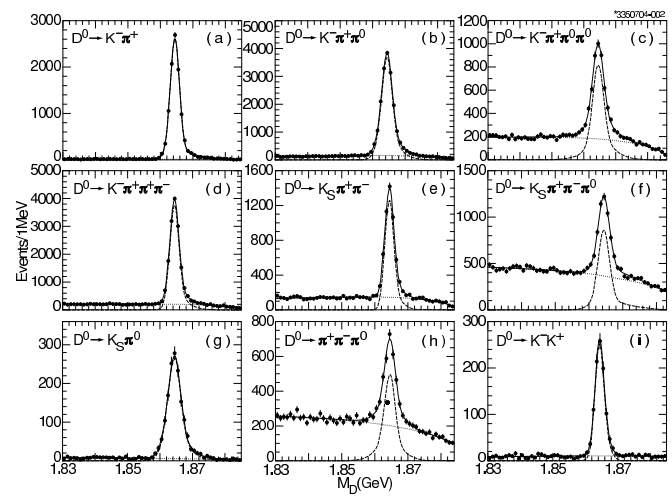

Figure 3. Fits to the beam-constrained masses for different fully reconstructed $D^{0}$ decay modes. The signal is described by a Gaussian and a bifurcated Gaussian to account for the initial state radiation. The background is described by an Argus function.

$K_{S} \pi^{+} \pi^{-}, K_{S} \pi^{+} \pi^{-} \pi^{0}, \pi^{+} \pi^{-} \pi^{0}$, and $K^{-} K^{+}$. Charge conjugate decays are implied. Within the tagged events, we select the subset in which the $\bar{D}^{0}$ meson semileptonically decays to a specific final state. The efficiencycorrected ratio of the event yields gives the absolute branching fraction for the exclusive semileptonic decay mode. The selection of the tag $D^{0}$ candidates is based on two variables $\Delta E=E_{D}-E_{\text {beam }}$ (the difference between the energy of the tag $D^{0}$ candidate $\left(E_{D}\right)$ and the beam energy $\left(E_{\text {beam }}\right)$ ), and the beam constrained mass $M_{b c}=$ $\sqrt{E_{\text {beam }}^{2}-p_{D}^{2}}$, where $p_{D}$ is the momentum of the tag $D^{0}$ candidate. Fits to the beamconstrained mass distributions for $D^{0}$ candidates are shown in Figure 3.

We then find $D^{0}$ semileptonic decays into $K^{-} \ell^{+} \nu, \pi^{-} \ell^{+} \nu, K^{*-} \ell^{+} \nu\left(K^{*-} \rightarrow K^{-} \pi^{0}\right)$, and $\rho^{-} \ell^{+} \nu\left(\rho^{-} \rightarrow \pi^{-} \pi^{0}\right)$ against a tag $D^{0}$ by reconstructing the difference of the missing energy and missing momentum which should peak at zero. In Figure 4, we present the $U$ distributions from data and $\mathrm{MC}$ for $D^{0} \rightarrow K^{-} \ell^{+} \nu, \pi^{-} \ell^{+} \nu, K^{*-} \ell^{+} \nu$ and $\rho^{-} \ell^{+} \nu$ The comparison shows good agreement between the data and MC.

The fits to the $U=E_{\text {miss }}-p_{\text {miss }}$ distributions are shown in Figure 5. For $D^{0} \rightarrow$
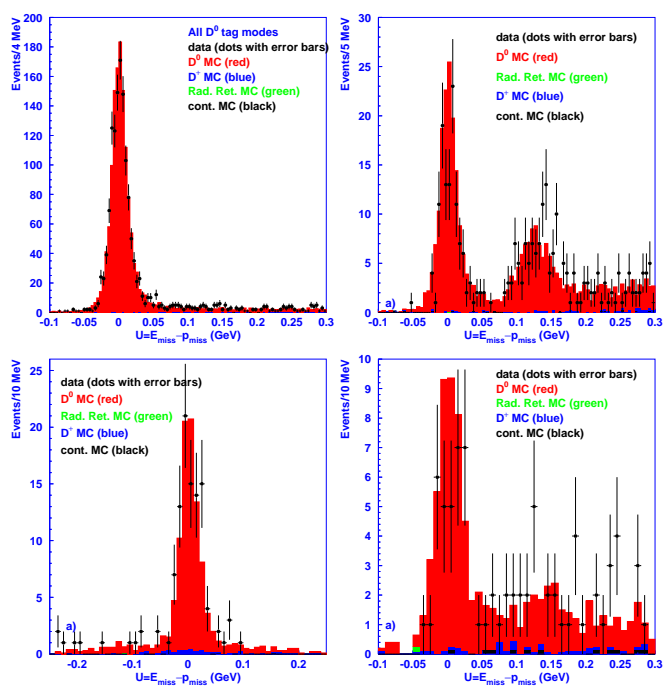

Figure 4. $U=E_{\text {miss }}-p_{\text {miss }}$ for $D^{0} \rightarrow K^{-} \ell^{+} \nu$ (top left), $\pi^{-} \ell^{+} \nu$ (top right), $K^{*-} \ell^{+} \nu$ (bottom left) and $\rho^{-} \ell^{+} \nu$ (bottom right) from data and MC.

$K^{-} \ell^{+} \nu$, the observed yield is $1405.1 \pm 38.5$ which corresponds to an absolute branching fraction measurement of $\mathcal{B}\left(D^{0} \rightarrow K^{-} \ell^{+} \nu\right)=$ $(3.52 \pm 0.10 \pm 0.25) \%$, comparing with $(3.58 \pm$ $0.18) \%$ from PDG. For $D^{0} \rightarrow \pi^{-} \ell^{+} \nu$, the observed yield is $109.1 \pm 10.9$ which corresponds to an absolute branching fraction measurement of $\mathcal{B}\left(D^{0} \rightarrow \pi^{-} \ell^{+} \nu\right)=(0.25 \pm 0.03 \pm$ $0.02) \%$, comparing with $(0.36 \pm 0.06) \%$ from PDG. For $D^{0} \rightarrow K^{*-} \ell^{+} \nu$, the observed yield is $88.0 \pm 9.7$ which corresponds to an absolute branching fraction measurement of $\mathcal{B}\left(D^{0} \rightarrow K^{*-} \ell^{+} \nu\right)=(2.07 \pm 0.23 \pm 0.18) \%$, comparing with $(2.15 \pm 0.35) \%$ from PDG. For $D^{0} \rightarrow \rho^{*-} \ell^{+} \nu$, the observed yield is $30.1 \pm 5.8$ which corresponds to an absolute branching fraction measurement of $\mathcal{B}\left(D^{0} \rightarrow\right.$ $\left.\rho^{*-} \ell^{+} \nu\right)=(0.19 \pm 0.04 \pm 0.02) \%$, this is the first observation and measurement of this decay mode. We also measure the ratio of absolute branching fractions to be: $\frac{\mathcal{B}\left(D^{0} \rightarrow \pi^{-} \ell^{+} \nu\right)}{\mathcal{B}\left(D^{0} \rightarrow K^{-} \ell^{+} \nu\right)}$ $=(7.0 \pm 0.7 \pm 0.3) \%((10.1 \pm 1.8) \%$ from PDG $)$, and $\frac{\mathcal{B}\left(D^{0} \rightarrow \rho^{-} \ell^{+} \nu\right)}{\mathcal{B}\left(D^{0} \rightarrow K^{*-} \ell^{+} \nu\right)}=(9.2 \pm 2.0 \pm 0.8) \%$.

The details of this work can be found in Ref. ${ }^{4}$. We can see that most of our measurements based on first $60 \mathrm{pb}^{-1}$ of CLEO- 


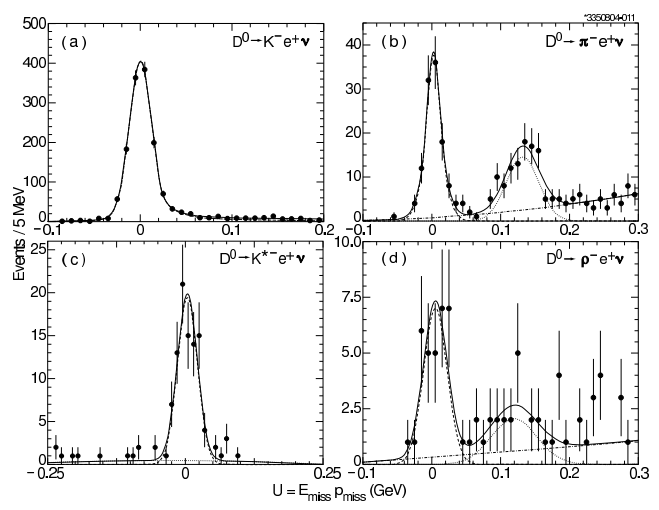

Figure 5. Fits to $U=E_{\text {miss }}-p_{\text {miss }}$ distributions for $D^{0} \rightarrow K^{-} \ell^{+} \nu, \pi^{-} \ell^{+} \nu, K^{*-} \ell^{+} \nu$ and $\rho^{-} \ell^{+} \nu$, with the other $\overline{D^{0}}$ fully reconstructed.

c data are already better than those listed in PDG. We also present the first observation of $D^{0} \rightarrow \rho^{-} \ell^{+} \nu$ decay. The errors are statistical and systematic, respectively. The dominant systematic error comes from uncertainties of track and $\pi^{0}$ reconstruction efficiency (3\% per track and $4.4 \%$ per $\pi^{0}$ ) which will improve with a larger data sample and further study. Our results for $D^{0} \rightarrow K^{-} \ell^{+} \nu$ and $D^{0} \rightarrow K^{*-} \ell^{+} \nu$ are consistent with those from the PDG. Our result for $\mathcal{B}\left(D^{0} \rightarrow \pi^{-} \ell^{+} \nu\right)$ is lower than the PDG value. The ratio $\frac{\mathcal{B}\left(D^{0} \rightarrow \pi^{-} \ell^{+} \nu\right)}{\mathcal{B}\left(D^{0} \rightarrow K^{-} \ell^{+} \nu\right)}$ is close to the CLEO III result $(8.2 \pm 0.6 \pm 0.5) \%^{2}$, while lower than the PDG value.

\section{References}

1. B. Grinstein, N. Isgur and M. B. Wise, Phys. Rev. Lett. 56 (1986) 258; F. J. Gilman and R. L. Singleton, Phys. Rev. D 41 (1990) 142; K. Hagiwara, A. D. Martin and M. F. Wade, Nucl. Phys. B 327 (1989) 569.

2. CLEO Collaboration, G.S. Huang et al., CLNS 04/1876, CLEO 04-06, CLEO CONF 04-14 and ICHEP04 ABS110780. Submitted to Phys. Rev. Lett.

3. S. Eidelman et al., Particle Data Group, Phys. Lett. B592, 1 (2004).
4. K. Y. Gao et al., CLEO Collaboration, hep-ex/0408077, contributed to the $32^{\text {nd }}$ International Conference on High Energy Physics (ICHEP'0 4), Beijing, August 16-22 2004. 